\title{
Improved working memory performance following administration of a single dose of American ginseng (Panax quinquefolius L.) to healthy middle-age adults
}

\author{
Anastasia Ossoukhova ${ }^{1}$, Lauren Owen ${ }^{2}$, Karen Savage ${ }^{1}$, Marjolaine Meyer $^{3}$, Alvin Ibarra ${ }^{4}$, Marc Roller ${ }^{5}$, \\ Andrew Pipingas ${ }^{1}$, Keith Wesnes ${ }^{1}$ and Andrew Scholey ${ }^{1}$ * \\ ${ }^{1}$ Centre for Human Psychopharmacology, Swinburne University, Melbourne, Victoria, Australia \\ ${ }^{2}$ Human Nutrition Unit, Department of Oncology, University of Sheffield, Sheffield, UK \\ ${ }^{3}$ Naturex Spain SL, Quart de Poblet, Spain \\ ${ }^{4}$ Naturex Inc., South Hackensack, New Jersey, USA \\ ${ }^{5}$ Naturex SA, France
}

\begin{abstract}
Objective A ginsenoside-rich extract of American ginseng (Panax quinquefolius L.), Cereboost ${ }^{\mathrm{TM}}$, was previously shown to improve working memory and mood in healthy young individuals. The present study represented a partial replication investigating whether these effects extended to healthy middle-aged individuals.

Methods Fifty-two healthy volunteers (40-60 years old, mean age 51.63) received $200 \mathrm{mg}$ of P. quinquefolius or a matching placebo according to a double-blind, placebo-controlled, balanced, crossover design. The Cognitive Drug Research battery and the Computerised Mental Performance Assessment System were used to evaluate cognitive performance at baseline then 1, 3 and $6 \mathrm{~h}$ following treatment. Blood glucose and mood were co-monitored.

Results Compared with placebo, P.quinquefolius improved cognitive performance on 'Working Memory' factor at $3 \mathrm{~h}$. Similar effects were observed in one of the two tasks making up this factor, spatial working memory. There were no significant effects on mood or blood glucose levels. Conclusions These data confirm that P.quinquefolius can acutely benefit working memory and extend the age range of this effect to middle-aged individuals. These changes are unlikely to be underpinned by modulation of blood glucose in this population. Copyright (C) 2015 John Wiley \& Sons, Ltd.
\end{abstract}

KEY WORDS—ginseng; working memory; cognitive enhancement

\section{INTRODUCTION}

Extracts of ginseng have been recognised for decades for their effects on the central nervous system. They have both stimulatory and inhibitory effects (Kennedy and Scholey, 2003; Smith et al., 2014) that may modulate neurotransmission, enhance memory and learning and provide neuroprotection. In a series of human trials, we have previously confirmed acute cognitive effects of Asian (Panax) ginseng (e.g. Kennedy et al., 2001a; 2001b; Scholey and Kennedy, 2002; Reay et al., 2005; 2006) and, more recently American ginseng Panax quinquefolius (Scholey et al., 2010).

The main active constituents in ginseng extracts are believed to be the triterpenoid saponins, known as ginsenosides (Attele et al., 1999). These can be classified into three groups on the basis of their chemical

*Correspondence to: A Scholey, Centre for Human Psychopharmacology, Swinburne University, Melbourne, VIC 3122, Australia. Tel: +61(3) 92148932; Fax: +61(3) 92145525. E-mail: andrew@ scholeylab.com structure; the Panaxadiol group ( $\mathrm{Rb} 1, \mathrm{Rb} 2, \mathrm{Rb} 3, \mathrm{Rc}$, etc.), the Panaxatriol group ( $\mathrm{Re}, \mathrm{Rf}, \mathrm{Rg} 1, \mathrm{Rg} 2$, Rh1) and the Oleanolic acid group (e.g. Ro). $P$. quinquefolius typically contains $4-6 \%(\mathrm{w} / \mathrm{w})$ total ginsenosides, with ginsenosides $\mathrm{Rb} 1, \mathrm{Re}$ and $\mathrm{Rd}$ being the most abundant (Qi et al., 2011). As with other herbal extracts, one challenge in this area relates to standardisation of treatment. It has been previously shown that there can be batch-to-batch variability in ginseng, with some extracts containing little or no ginsenosides (Harkey et al., 2001; Sievenpiper et al., 2004). When it comes to investigations of herbal substances in the psychopharmacology domain, it has been suggested that absence of the standardised herbal products on the market might have delayed the progress and consistency of the research in this area considerably and implementation of the standardised products provided a platform for systematic scientific investigations (Scholey et al., 2005). Indeed, consistency of cognitive results 
obtained with Panax ginseng to some extent is due to the availability of its highly standardised extract (G115); hence, the availability of a standardised $P$. quinquefolius extract $\left(\right.$ Cereboost ${ }^{\mathrm{TM}}$ ) also provides an opportunity to commence a systematic assessment of its neurocognitive effects.

Cereboost ${ }^{\mathrm{TM}}$ is a dry native extract of $P$. quinquefolius L. roots with a high ginsenoside content, standardised to $10-12 \%$ total ginsenosides (Scholey et al., 2010). This is up to threefold greater total ginsenosides than the $P$. ginseng extract G115. We recently reported findings of the randomised, double-blind, placebocontrolled, balanced, crossover trial evaluating the acute mood, neurocognitive and blood glucose effects of 100,200 and $400 \mathrm{mg}$ of Cereboost ${ }^{\mathrm{TM}}$ in young adults (Scholey et al., 2010). The extract was associated with a significant and consistent improvement to working memory performance. Spatial working memory, assessed using Corsi blocks, was improved by all doses at all testing times (1, 3 and $6 \mathrm{~h}$ post treatment). There were also significant improvements to numeric working memory speed and immediate word recall associated with $200 \mathrm{mg}$ dose. Alphabetic working memory speed was enhanced by 100 and $400 \mathrm{mg}$ doses. There were also a number of time-specific and task-specific benefits associated with other doses. Self-rated calmness was increased following $100 \mathrm{mg}$ dose at 3 and $6 \mathrm{~h}$ post treatment. Although potent glycaemic properties of $P$.quinquefolius has been previously reported (Vuksan et al., 2000a, 2000b, 2000c), no treatmentrelated changes in glucose levels were observed in the previous study. Given that the glycaemic effects of $P$. quinquefolius (Vuksan et al., 2000c) and the cognitive impact of blood glucose changes (for example a slightly older, middle-aged cohort, Messier et al., 2010), may be more pronounced in individuals with poorer glucoregulation, blood glucose was comonitored in the 40 to 60 -year-old volunteers in the current study.

Normal ageing is associated with neurocognitive decline, which is likely underpinned by numerous systemic and central structural and functional changes. These include changes to neuronal connectivity, cellular calcium regulation, protein formation and destruction (proteolysis), neurotransmitter and hormonal changes and cerebral blood flow (Bishop et al., 2010). Because these numerous biological changes lead to impairments during the course of ageing, it is likely that greater scope for cognitive enhancement exists in older populations. For example, the effects of other herbal extracts such as sage are greater in older than younger individuals (Scholey et al., 2008), as are the effects of glucose administration (e.g. Macpherson et al., 2014). Therefore, a logical step in evaluating the cognition-enhancing potential of pharmaceuticals, or nutraceuticals such as P.quinquefolius is to evaluate their effects in a slightly older cohort including, initially, with acute dosing.

The Cognitive Drug Research (CDR) battery has a large database of normative data, which indicates that working memory begins to decline in middle age (Wesnes, personal communication). The battery has been used in approximately 1400 clinical trials - many involving the cognitive and mood effects of nutraceutical interventions, including $P$. ginseng (Kennedy et al., 2001a, 2001b; 2002). The current study therefore utilised the CDR battery in order to allow direct comparison with these studies. Because our previous study found positive effects of P.quinquefolius in healthy young adults on selected tasks form the Computerised Mental Performance Assessment (COMPASS) battery, these tasks were also included to gauge whether similar effects would be observed on the same tasks in a slightly older cohort. The use of both batteries allowed cross-platform comparison of the two assessment systems. The current study therefore specifically aimed to extend previous findings by assessing the cognitive effects of a single dose of P.quinquefolius (200 mg encapsulated Cereboost ${ }^{\mathrm{TM}}$ ) selected on the basis of previous findings (Scholey et al., 2010) on aspects of working memory processes in a slightly older group of middle-aged (40-60 years) healthy volunteers. Specifically, we hypothesised that the administration of Cereboost ${ }^{\mathrm{TM}}$ would be associated with improved working memory performance in this population.

\section{MATERIALS AND METHODS}

\section{Design}

The investigation was a single centre, double-blind, placebo-controlled, balanced, two-period, crossover design study conducted at the Centre for Human Psychopharmacology, Swinburne University, Australia. The trial was registered on the Australian New Zealand Clinical Trials Registry as ACTRN12610000849099.

\section{Participants}

A power calculation produced an $\mathrm{N}$ of 46 to detect a significant change with a power of 0.8 at the 0.005 alpha levels (adjusted from 0.05 to allow for multiple comparisons). As a result, there was a requirement to recruit at least 50 participants to allow for dropout.

Fifty-four volunteers (22 male and 32 female volunteers, mean age 51.63 , SD 6.35 years) took part in this study approved by the Swinburne University Human 
Research Ethics Committee and conducted according to the Declaration of Helsinki. All participants gave written informed consent and received AU \$200 for their participation upon the completion of the study.

Participants completed a health screening questionnaire, and all reported that they were in good health, not taking any drugs or medications (excluding the contraceptive pill), had no known food allergies and were non-smokers. Participants with a number of medical conditions such as diabetes, hypoglycaemia, psychiatric disorders, epilepsy and gastrointestinal disorders, who were taking prescribed medications, were pregnant or lactating were excluded from the study. They were screened for cognitive decline using the Mini-Mental State Examination (Folstein et al., 1975), and all had scores of 27 and above, indicating that they were cognitively intact. Subjects also completed the Depression Anxiety and Stress Scale (Lovibond and Lovibond, 1995) to confirm absence of the three negative affective states of depression, anxiety and stress.

Enrolled participants refrained from taking any vitamins, other herbal supplements and over the counter medicines for the period of the study. On the testing days, participants also abstained from consuming alcohol, caffeine products and energy drinks.

Two participants withdrew from the study (one for reasons unrelated to the study and one due to an adverse event (AE) remotely related to the study product). These volunteers were excluded from analysis, giving a total sample size of 52 participants.

\section{Treatments}

Commercial extract of P.quinquefolius Cereboost ${ }^{\mathrm{TM}}$ provided by Naturex (Naturex, Avignon, France) was prepared according to methods described by Scholey et al. (2010). According to analysis of ginsenosides by high-performance liquid chromatography performed by a manufacturer, Cereboost ${ }^{\mathrm{TM}}$ was standardised to $11.65-11.67 \%$ ginsenosides with individual ginsenoside content as follows: Rb1 (5.69\%), $\operatorname{Re}(2.06 \%), \operatorname{Rc}(1.87 \%), \operatorname{Rd}(1.48 \%), \operatorname{Rb} 2(0.29 \%)$ and $\operatorname{Rg} 1(0.28 \%)$. Cellulose powder (Avicel ${ }^{\circledR} \mathrm{PH}$ Microcrystalline Cellulose, FMC BioPolymer, PA) served as a placebo. Encapsulation was conducted at Thompsons Amcal Pharmacy in Melbourne, Australia. Treatments were soft, opaque, gelatine-based capsules of identical appearance, taste and smell. In order to adhere to double-blind methodology standards, each individual's treatments were prepared by a disinterested third party who took no further part in the study. On each of the two study days, participants received one capsule containing either $200 \mathrm{mg}$ of Cereboost ${ }^{\mathrm{TM}}$ or placebo, depending on the condition to which they were allocated on that particular day. Counterbalanced treatment allocation was performed by computer-generated randomisation.

\section{Cognitive and mood measures}

Cognitive Drug Research computerised assessment battery. The CDR computerised assessment battery has been used in more than 1400 drug and nutritional interventional trials. The tailored version of the CDR battery utilised here, including a description of the constituent tasks, has been described in detail previously (Scholey and Kennedy, 2004; Scholey et al., 2008). This battery has previously been found to be sensitive to modulation of cognitive function as a consequence of acute ingestion of $P$. ginseng (Kennedy et al., 2001b; 2002), Ginkgo biloba (Kennedy et al., 2000; 2001a; 2002; 2007) and a Ginkgo biloba/ P. ginseng combination (Kennedy et al., 2001a).

The selection of computer-controlled tasks was administered in fixed order with parallel versions of the tasks randomly presented between participants at each testing session. Presentation was via highresolution computer monitors and, all responses were recorded via two-button (yes/no) response boxes with the exception of written word recall tests. The tasks were presented as follows: Word presentation, immediate word recall, picture presentation, simple reaction time, digit vigilance, choice reaction time, spatial working memory, numeric working memory, delayed word recall, word recognition and picture recognition. In all, CDR battery tasks took approximately $20 \mathrm{~min}$ to complete. Detailed description of the tasks can be found in previous publications (Kennedy et al., 2000; 2001a; 2002; 2007; Pengelly et al., 2012; Wesnes et al., 2000; Sunram-Lea et al., 2005).

As with previous studies assessing $P$.ginseng, the single task outcomes from the CDR battery were collapsed into the five cognitive outcome factors derived by a factor analysis as previously described by Wesnes et al. (2000) and presented graphically by Scholey and Kennedy (2004). The factor composition is briefly outlined in the following text.

'Working Memory' factor: derived by combining the sensitivity index scores from the two working memory tests-spatial working memory and numeric working memory. $100 \%$ accuracy across the two tasks would generate a maximum score of 2 on this index.

'Secondary Memory' factor: derived by combining the percentage accuracy scores from delayed word recognition, delayed picture recognition, immediate word recall and delayed word recall tasks. Accuracy 
of $100 \%$ across the four tasks would generate a maximum score of 400 on this index.

'Speed of Memory' factor: derived by combining the reaction times of numeric working memory, spatial memory, delayed word recognition and delayed picture recognition (units are summed milliseconds from the four tasks).

'Speed of Attention' factor: derived by combining the reaction times of the three attentional taskssimple reaction time, choice reaction time and digit vigilance (units are summed milliseconds for the three tasks).

'Accuracy of Attention' factor: derived by calculating the combined percentage accuracy across the choice reaction time and digit vigilance tasks. Accuracy of $100 \%$ across the two tasks would generate a maximum score of 100 .

'Speed of Attention' and 'Accuracy of Attention' factors were described as 'Power of Attention' and 'Continuity of Attention" in the original factor analysis by Wesnes et al. (2000); however, description utilised in this research has been implemented in previous research on the effects of herbal extracts on cognition (e.g. Kennedy et al., 2002; Sunram-Lea et al., 2005; Haskell et al., 2007).

Computerised Mental Performance Assessment System battery. The COMPASS testing battery has been developed to include tests, which have been shown to be sensitive to nutritional manipulations including to acute administration of American ginseng, and tasks are described in detail by Scholey et al. (2010). Parallel versions of each of the following tasks allowed for multiple testing. This suite of tasks took approximately $10 \mathrm{~min}$ to complete, and they were presented as follows:

Alphabetic working memory: A series of five letters were presented on the computer screen for $4 \mathrm{~s}$ for participants to memorise. This was followed by a series of 30 probe letters. Participants had to indicate whether the letter was from the original series pressing computer keyboard keys corresponding to YES or NO as quickly as possible. The task was scored for accuracy (\%) and speed (ms) of performance.

Serial 7: Participants were required to count backwards in sevens from a random starting number between 800 and 999, presented on the computer screen, as quickly and accurately as possible. Participants were using the numeric key pad to enter each three-digit response. After the first subtraction was entered via the numeric key pad, each digit was replaced on the screen by an asterisk. The task was scored for a number of measures: total number of generated subtractions (n), number of correct (n) as well as incorrect (n) subtractions, average accuracy $(\%)$ and speed (ms) of performance, which evaluated average time taken participant to complete one subtraction (ms). In case of incorrect responses, the subsequent responses were scored as positive if they were correct in relation to the new number.

RVIP: A series of digits were presented on the computer screen, one at a time, at the rate of 100 digits per min in quick succession. Participants monitored continuous series for the target sequences: they were required to detect consecutive sequences of three even (combination of the following 2-4-6-8) or odd (combination of the following 1-3-5-7-9) numbers and asked to respond by pressing a space bar as soon as a target string was detected. Duration of the task was 3 min with eight correct target strings presented per minute. The task was scored for accuracy of performance (\% of correctly identified target strings), the average reaction time (ms) to the correctly identified sequences, and the number (n) of false alarms. Corsi Blocks: In all nine identical blue blocks (size $93 \times 93$ pixels) were presented dispersed randomly on the computer screen. A predetermined number of blocks were illuminated (briefly changed colour to red) sequentially at the rate of one per second identifying a sequence of spatial locations to be remembered. Volunteers repeated presented sequence by clicking boxes on the screen pointing cursor and clicking computer mouse. Task difficulty progressively increased increasing number of the presented blocks from four upwards (maximum of nine sequentially illuminated blocks). The task outcome (score) was a measure of spatial span, which reflected maximum of squares participants were able to repeat correctly.

Visual analogue mood scales: The computerised version of Bond-Lader Mood scales (Bond and Lader, 1974) was incorporated within a number of the selected COMPASS tasks. In all, 16 mood scales, comprised of $100 \mathrm{~mm}$ visual analogue scales anchored at each end by pairs of mood antonyms (e.g. calm-tense). These were combined as recommended by the authors to form three mood factors: 'alert', 'calm' and 'content'. Two other visual analogue scales assessed stress and mental fatigue and were described previously by Scholey et al. (2010). Briefly, participants were required to place a mark along the line of the visual analogue scale with end points anchored by 'not at all' and 'extremely" in response to the questions 'How (stressed/mentally fatigued) do you feel right now?' Scores ranged 
from 1 to 100 with higher scores reflecting higher subjective feelings of stress/mental fatigue.

\section{Pencil-and-paper questionnaires}

Depression Anxiety and Stress Scales (DASS): The shortened 21-item version of the DASS (Lovibond and Lovibond, 1995) was used to assess three negative affective states of depression, anxiety and stress on seven-item scales on the practice day. The Depression subscale (DASS-D) measures symptoms relating to dysphoric mood (e.g. sadness), for example 'I couldn't seem to experience any positive feeling at all'. The Anxiety subscale (DASS-A) assesses symptoms associated with physiological hyperarousal such as autonomic arousal, for example 'I felt I was close to panic'. The Stress subscale (DASS-S) assesses symptoms associated with nervous arousal, for example 'I tended to over-react to situations'.

Mini-Mental State Examination is a standard tool for screening cognitive impairments (Folstein et al., 1975). It is a brief 30-point questionnaire test, which facilitates the detection of mental status changes, especially in older cohorts, and frequently used to verify absence of cognitive decline (Folstein, 2007). The Mini-Mental State Examination was completed during the practice day as a part of screening procedure with a score equal or greater than 24 as an inclusion criterion.

Profile of Mood States (POMS) is a self-report questionnaire designed to measure six dimensions of mood: tension-anxiety; depression-dejection; anger-hostility; vigour-activity; fatigue-inertia; and confusionbewilderment). The Profile of Mood States consists of 65 adjectives describing feeling and mood which is answered on a five-point Likert-type scale ranging from not at all to extremely. Respondents are asked to indicate mood reactions for the 'past week including today' or for shorter periods such as 'right now.' This questionnaire was completed at the beginning of the testing days to identify whether there were differences in participants' mood at the start of the day (before the baseline assessments and subsequently treatment administration), which could have potentially influenced the levels of cognitive performance during the day.

State-Trait Anxiety Inventory (STAI) comprised of two scales. The 'State' (STAI-S) subscale is widely used for measuring fluctuating levels of anxiety. The subscale contains 20 statements regarding current mood (e.g. 'I am calm'). Participants rate how much they feel like each statement at the time of making the response by marking a 4-point scale ranging from 'not at all' to 'very much so'. The 'Trait' (STAI-T) subscale comprises 20 different statements (e.g. 'Some unimportant thought runs through my mind and bothers me'). Participants were asked to indicate how they generally feel on a scale ranging from 'almost never' to 'almost always'. Scores on both sections of the STAI range from 20 to 80, with higher scores indicating more anxiety. Participants completed this scale at the beginning of each visit to ensure that their anxiety levels did not differ prior to the commencement of the assessments and administration of treatments.

Leeds Sleep Evaluation Questionnaire (SEQ) comprises of 10 self-rating 100 -mm-line analogue questions concerned with aspects of sleep and early morning behaviour. The questionnaire has been used to monitor subjectively perceived changes in sleep during psychopharmacological investigations. SEQ contains ten questions pertaining to four aspects of sleep: getting to sleep, quality of sleep, awakening from sleep, and behaviour following wakefulness. This questionnaire was completed at the beginning of each visit prior to the cognitive and mood assessments and administration of treatments to ensure that the quality of the previous night's sleep have not influenced participants' performance.

Blood glucose measures. As a part of the screening procedure, fasting blood glucose measurements were taken at the beginning of the practice day as well as each testing day prior to breakfast intake to ensure participants fasting blood glucose was $<6.1 \mathrm{mmol} / \mathrm{l}$. This value was chosen as the top end of a healthy fasting range as recommended by the local Diabetes Victoria (www.diabetesvic.org.au), also refer to Alberti and Zimmet (1998). On each of the two active study days, blood glucose levels were measured via capillary finger prick at a baseline (before a baseline assessment), then $1 \mathrm{~h}$ (before the commencement of the first posttreatment assessment), $3 \mathrm{~h}$ (before the commencement of the second post-treatment assessment) and $6 \mathrm{~h}$ (before the commencement of the third) post-treatment assessment following treatment administration. Blood glucose levels were monitored using a MediSense Optium Xceed Blood Glucose Sensor and disposable MediSense Blood Glucose Electrodes (MediSense Britain Ltd, Birmingham, UK). The accuracy and consistency of MediSense blood glucose sensors has previously been established (e.g. (Matthews et al., 1987). The reliability of the test has previously been confirmed (Price and Koller, 1988). Blood samples were taken using Owen Mumford 'Unistik 2' single use capillary blood sampling devices (Owen Mumford Ltd, Oxford, UK). Alcohol-soaked skin cleansing swabs was used for pre-sampling sterilisation. 
Blood pressure and heart rate measurement. Participants were seated calmly in a comfortable relaxed position for at least $10 \mathrm{~min}$ prior to the procedure. Blood pressure was then measured with an automated blood pressure sphygmomanometer, making sure that an arm with a fastened blood pressure cuff was at participants' heart level. Systolic blood pressure was expected to be between 91 and $139 \mathrm{mmHg}$ and diastolic blood pressure between 61 and $89 \mathrm{mmHg}$ and only participants whose blood pressure lay within normal ranges were included in the study. Heart rate was also recorded; this measure was not used for screening purposes.

Tolerability. Tolerability was assessed via monitoring of unsolicited AEs ('How have you been feeling'). To elicit reports of adverse events and inter-current illnesses, participants were required to complete the Symptom Checklist questionnaires at the beginning (Weekly Symptom checklist) and the end of each test session (Daily Symptom checklist). The symptom checklist was developed at the Brain Sciences Institute specifically for use with natural medicines and consists of 28 physiological/psychological problems people might have, for example, 'I feel dizzy', 'I have a dry mouth' and 'I feel anxious more than usual' (Stough et al., 2001). Participants indicated how much the problem had bothered them in the last 7 days including the day of recording using a 5-point scale from 'not at all' to 'very much so'.

\section{Procedure}

Each participant was required to attend the testing labs on a total of three occasions (one practice and two study days), conducted seven days apart to ensure sufficient wash-out between conditions. Testing took place in a suite of dedicated laboratories at the Centre for Human Psychopharmacology with participants visually isolated from each other. Participants were also wearing headphones to minimise distraction.

At the beginning of the first visit, participants signed their informed consent and then underwent a health screen procedure during which their morphometric and demographic data were collected and blood pressure, heart rate and fasting blood glucose were measured. Based on the outcomes of the health screen, participants eligible for the trial were familiarised with the protocol and procedures of the study. Additionally, participants filled in pen-and-paper questionnaires and completed three cycles of the computerised tasks of CDR and COMPASS batteries to minimise practice effects. Performance data from the practice session were not included in the analysis.
On arrival at their first testing visit, participants were randomly allocated to the treatment order determined by a computer-generated matrix. Fasting blood glucose was measured at the beginning of each visit to ensure that participants fasted before attending sessions, which was followed by standard light breakfast (piece of toast or cereal) consumed at least $1 \mathrm{~h}$ before the onset of the experiment. During this hour, prior to baseline assessment, they completed selected pen-andpaper questionnaires (STAI-S, POMS and Leeds SEQ) and later underwent four identical cognitive and mood assessments using parallel versions of the CDR battery as well as parallel versions of selected tasks from the COMPASS battery. Entire selection of the computer tasks (CDR and COMPASS) took approximately $30 \mathrm{~min}$ to complete. The first was a pre-dose testing session, which established baseline performance for the day, was immediately followed by the day's treatment (P.quinquefolius or placebo). Further testing sessions began at 1, 3 and $6 \mathrm{~h}$ following the consumption of the treatment. A standard light lunch was provided upon completion of $1 \mathrm{~h}$ post-dose assessment (between 1 and $3 \mathrm{~h}$ cognitive and mood assessments or approximately $1.5 \mathrm{~h}$ post-dose), which consisted of sandwich (with either chicken and salad, or cheese and salad), the same foods were consumed by each participant on each study day. Each assessment was preceded by blood glucose measurements (see Blood glucose measurements for details). The symptom checklist was completed at the end of the final testing session of the day to make sure that it was safe for participants to leave testing laboratories.

\section{Statistical analysis}

Only the data of the participants who attended all three visits and had a suitable level of compliance with all

Table 1. Demographic and morphometric characteristics of the volunteers $(\mathrm{N}=52)$

\begin{tabular}{lcccc}
\hline Measure & Mean & SD & Min & Max \\
\hline Age (years) & 51.63 & 6.47 & 40 & 60 \\
Education (years) & 16.60 & 3.04 & 11 & 25 \\
Height (cm) & 169.65 & 10.94 & 147 & 196 \\
Body weight (kg) & 71.15 & 14.23 & 45 & 110 \\
Body mass index (kg/m ${ }^{2}$ ) & 24.5 & 2.9 & 18.7 & 30 \\
Systolic blood pressure (mmHg) & 126.00 & 11.36 & 99 & 139 \\
Diastolic blood pressure (mmHg) & 78.2 & 8.5 & 63 & 89 \\
Heart rate (bpm) & 68.98 & 10.94 & 51 & 99 \\
Fasting blood glucose (mmol/L) & 5.04 & 0.52 & 4.1 & 6.00 \\
MMSE (score) & 29.23 & 0.81 & 27 & 30 \\
DASS (score) & 27.35 & 4.26 & 21 & 39 \\
STAI-trait (score) & 35.85 & 9.34 & 21 & 47 \\
\hline
\end{tabular}

MMSE, Mini-Mental State Examination; DASS, Depression Anxiety and Stress Scales; STAI, State-Trait Anxiety Inventory.

Mean scores are presented with standard deviations and ranges. 
Table 2. Effects of P. quinquefolius (Cereboost ${ }^{\mathrm{TM}}$ ) on individual task outcome measures from the Cognitive Drug Research battery

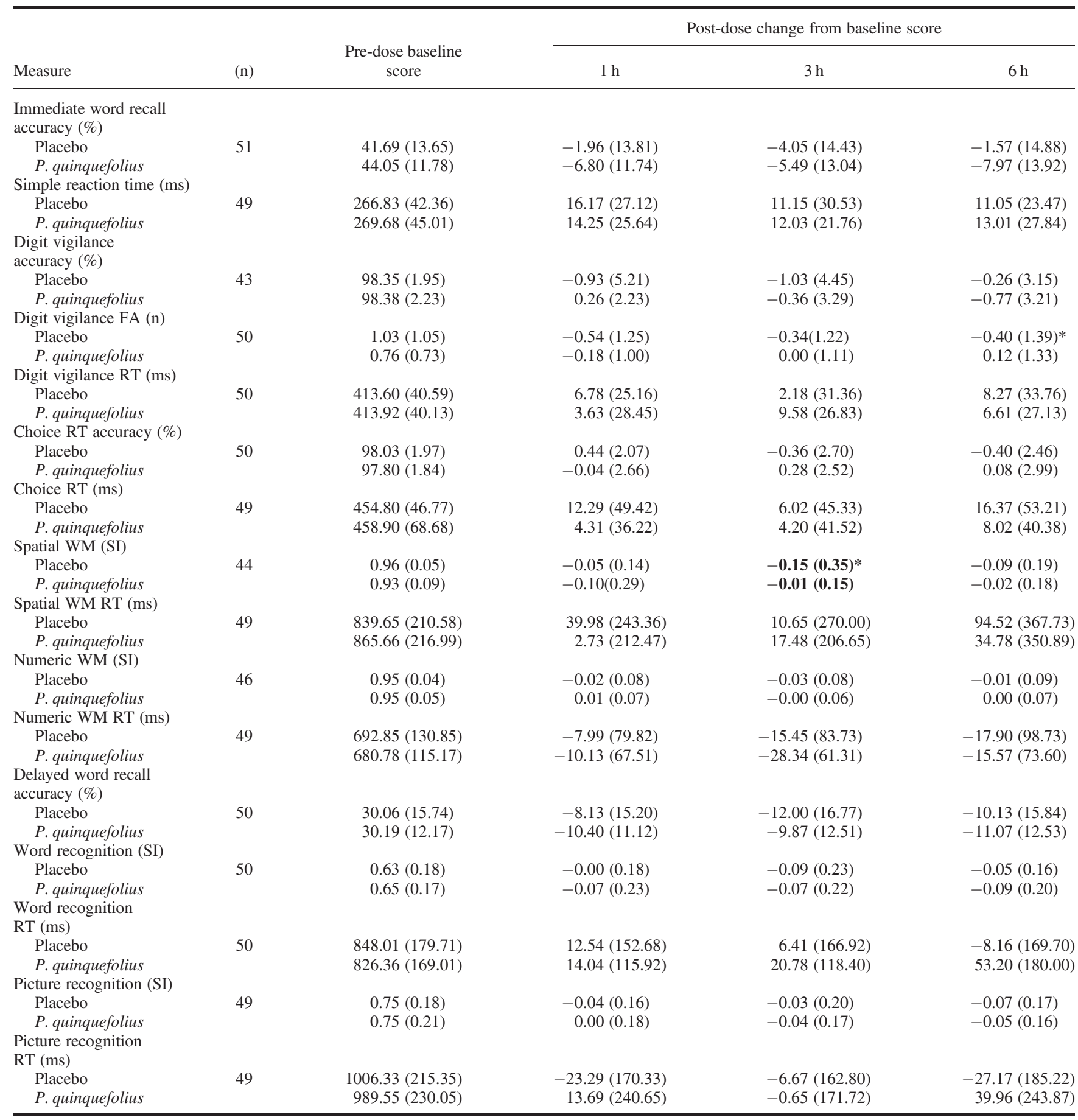

Mean baseline and change from baseline scores are presented with standard deviations in parentheses. $p$ - values between P. quinquefolius and placebo with Bonferroni correction are indicated $(*,<0.05)$.

study protocol requirements were selected for analyses. Across the participants, screening for univariate outliers revealed that scores for each outcome measure were normally distributed. Data points with mean scores more than three standard deviations outside the sample means were excluded from the analyses; therefore, a number of participants vary between the tasks depending on their performance on particular 
Table 3. Effects of P. quinquefolius (Cereboost ${ }^{\mathrm{TM}}$ ) on cognitive factors derived from the Cognitive Drug Research battery

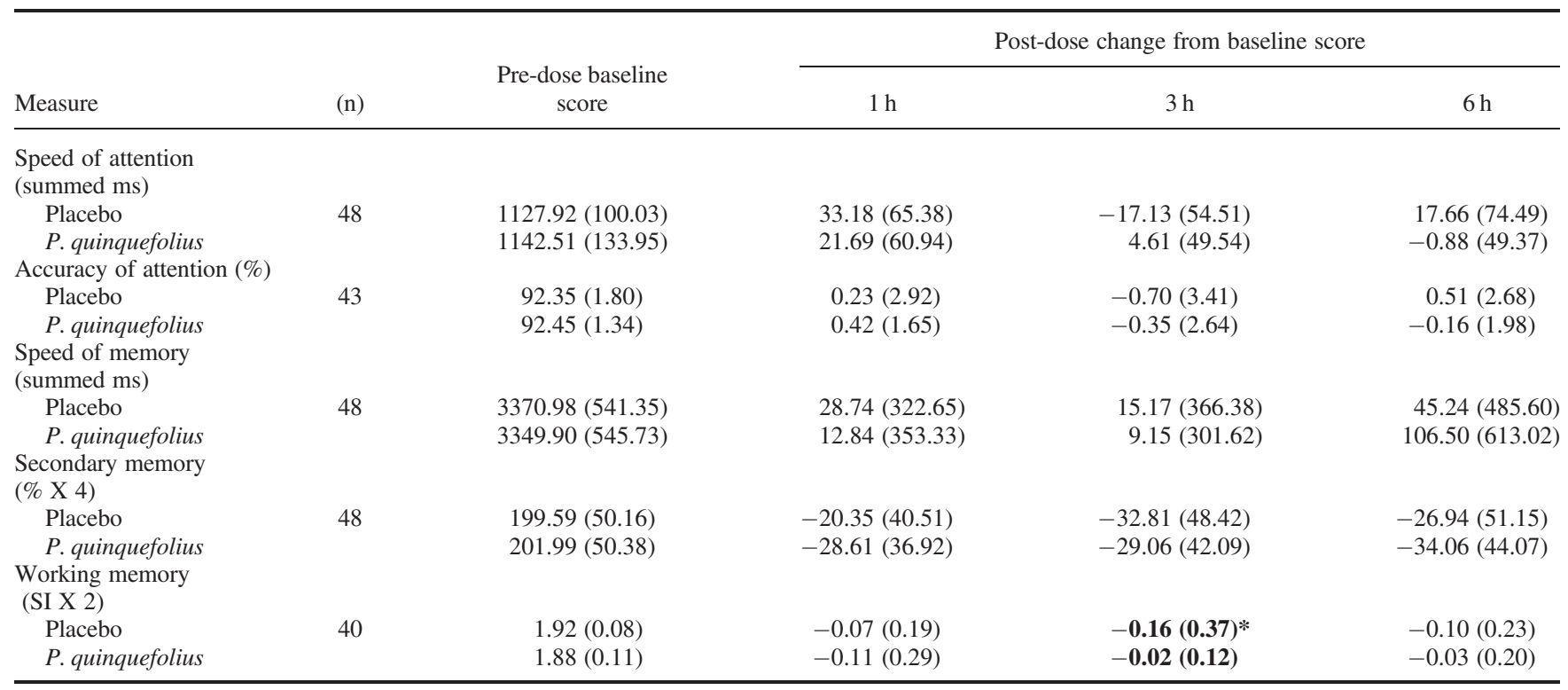

Mean baseline and change from baseline scores are presented with standard deviations in parentheses. $p$-values between P. quinquefolius and placebo with Bonferroni correction are indicated $(*,<0.05)$
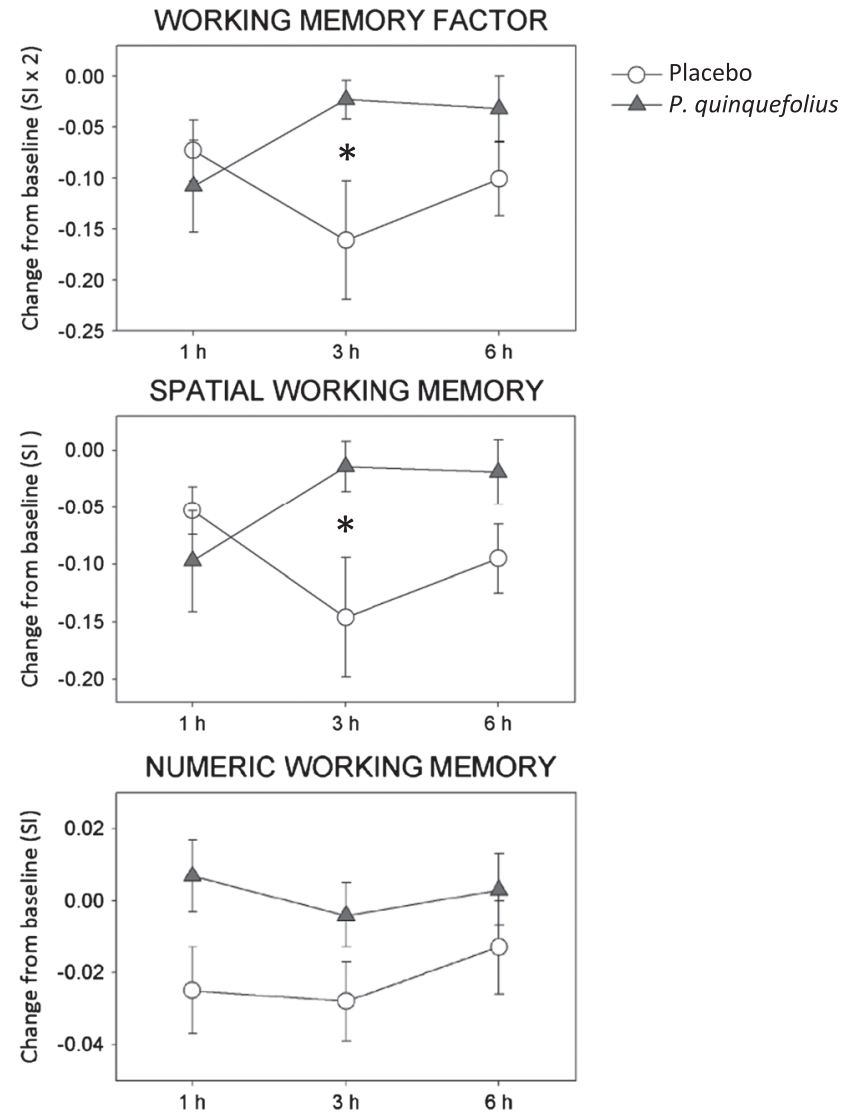

Figure 1. Effects of P. quinquefolius (Cereboost ${ }^{\mathrm{TM}}$ ) on working memory measures. Graphs depict mean scores with SEM 1, 3 and $6 \mathrm{~h}$ following a single dose of P.quinquefolius and placebo. Significant differences $(*,<0.05)$ at each time point are indicated measure. This especially evident in relation to Factor scores, which are composite scores based on performance not one but a number of tasks; however, it was confirmed that the treatment order was not affected by the removal of the outliers.

Scores from the pencil-and-paper questionnaires (POMS, Leeds SEQ and STAI-S) administered prior to the baseline assessment to ensure there were no in participant emotional and physical state between 2 days prior to treatment administration, which could affect cognitive performance and mood, were analysed with a one-way repeated-measures analysis of variance (ANOVA).

Scores on all CDR individual task outcomes, the five primary factors, the selected tasks from COMPASS battery outcomes and blood glucose levels were analysed as 'change from baseline' using the SPSS 17.0 statistical package for Windows. Prior to analysis of 'change from baseline' data, raw baseline scores for all these measures were subjected to a one-way repeated-measures ANOVA comparing placebo with $P$.quinquefolius to ensure there were no differences of performance between the study days. Next step, change-from-baseline scores were subjected to a twoway repeated-measures ANOVA with factors being 'treatment' (Cereboost ${ }^{\mathrm{TM}}$, placebo) and 'time' (1, 3 and $6 \mathrm{~h}$ ) with repeated measures on both factors. For this initial ANOVA, all testing was two-tailed at 5\% significance level. Bonferroni corrected paired contrasts were made comparing P.quinquefolius with 
Table 4. Effects of P. quinquefolius (Cereboost ${ }^{\mathrm{TM}}$ ) on individual task outcome measures from the Computerised Mental Performance Assessment battery

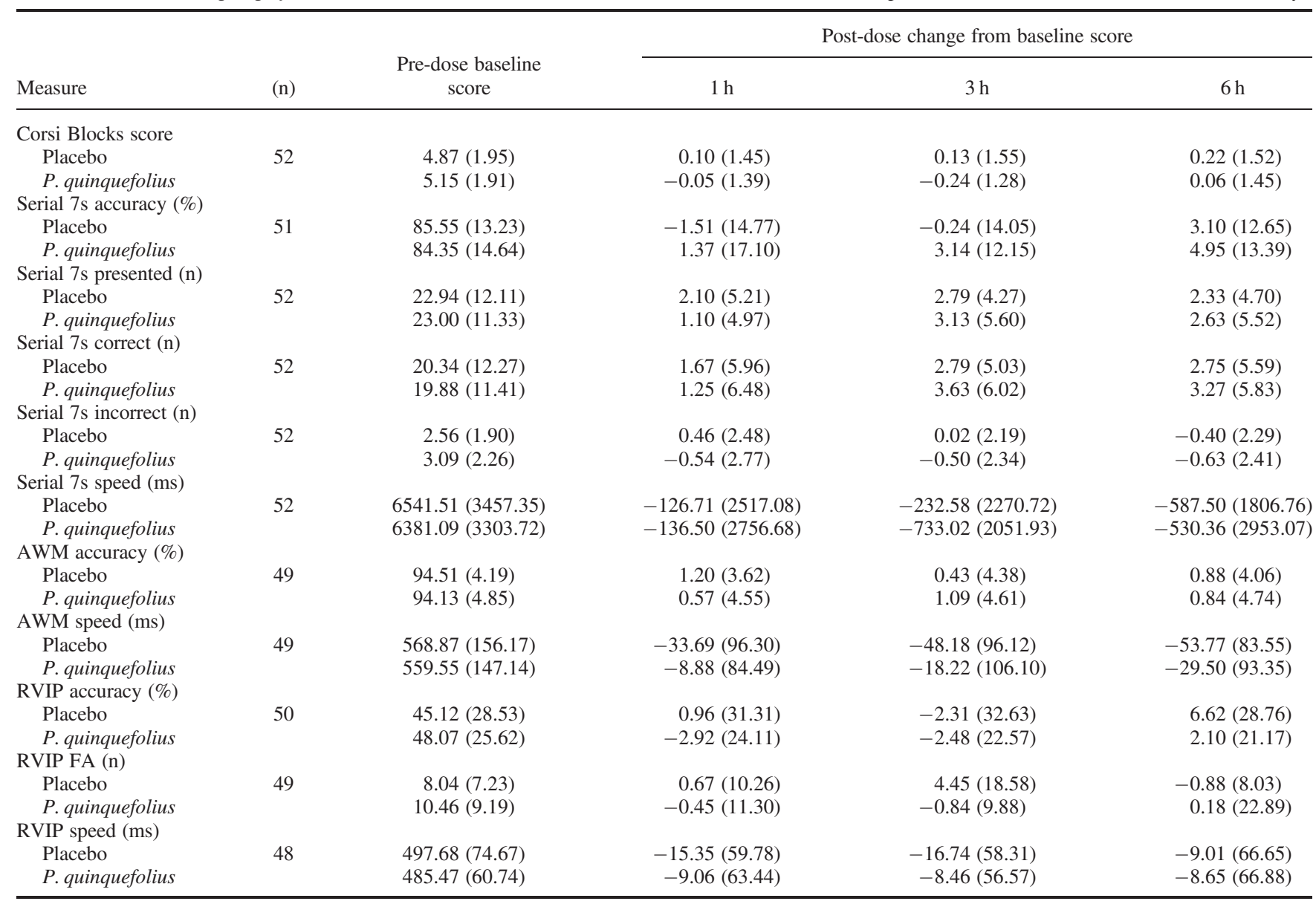

Mean baseline and change from baseline scores are presented with standard deviations in parentheses.

placebo at each time point. To ensure the overall Type I error protection level, only comparisons associated with measures that generated a significant main effect of Treatment, or Treatment $\times$ Time interaction on the initial ANOVA are reported, along with associated significant and trend differences between treatments at each time point.

\section{RESULTS}

\section{Participant characteristics}

Demographic and morphometric characteristics of the participants are summarised in Table 1 . Of the 52 participants who completed the trial $22(42.3 \%)$ were male, $44(84.6 \%)$ were right-handed and eight (15.4\%), 24 (46.2\%) and 20 (38.5\%) had secondary, tertiary and postgraduate education, respectively.

\section{Baseline scores}

According to a one-way repeated-measures ANOVA analysis of all computerised and pencil-and-paper scores comparing placebo with P.quinquefolius, there were no significant differences on any measure between the two conditions prior to treatment administration.

\section{Cognitive Drug Research individual tasks outcome measures}

Mean pre-dose baseline raw scores and change from baseline scores, for each treatment at each post-time point on the individual tasks are presented in Table 2. Where appropriate, the results of the planned comparison of individual task outcomes that generated a significant result on the initial ANOVA are described in the succeeding text in relation to the overall factor to which they contribute.

\section{Cognitive factors}

Mean raw baseline scores and change from baseline scores for each condition across each session are presented in the table and graphs of Table 3 and Figure 1. 
Table 5. Effects of P. quinquefolius (Cereboost ${ }^{\mathrm{TM}}$ ) on the mood factors derived from the Bond-Lader mood scales (Alert, Content and Calm) and on the 'mental fatigue' and 'stress' Visual Analogue Scales mood scores

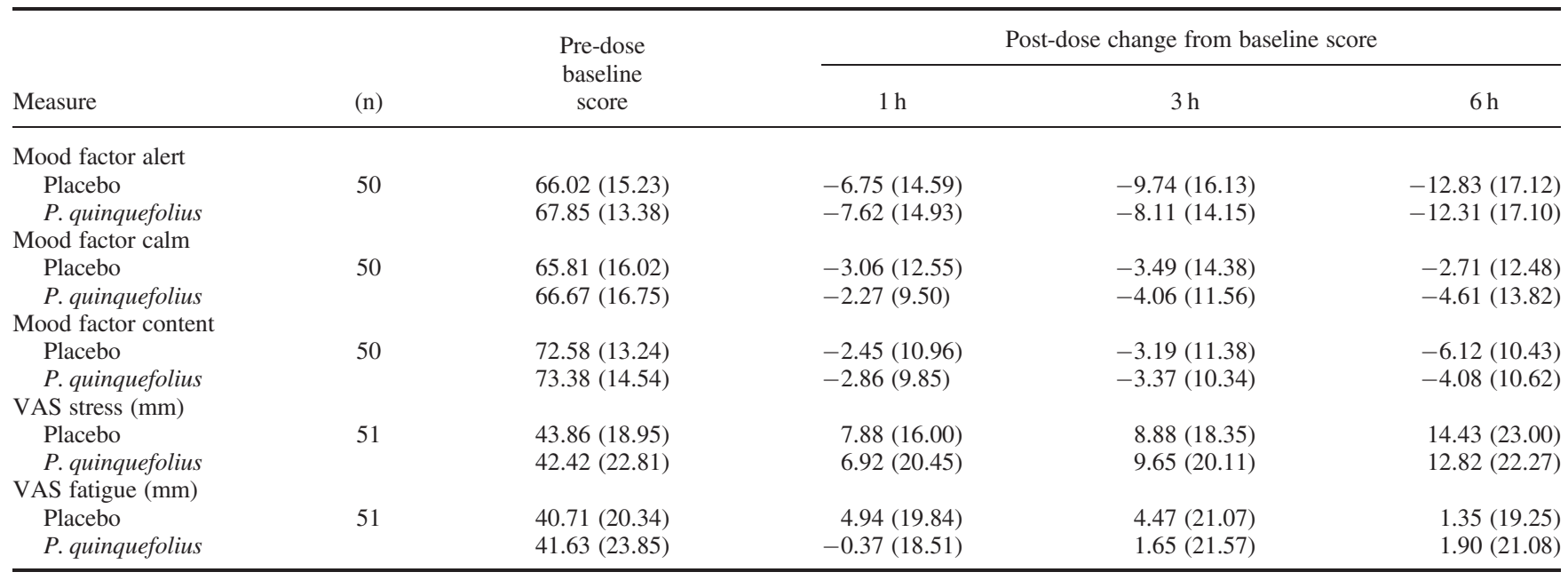

Mean baseline and change from baseline scores are presented with standard deviations in parentheses.

Working memory factor: The working memory factor was the primary outcome. The initial ANOVA showed a significant treatment $\mathrm{x}$ time interaction $[F(2,78)=3.380, p=0.039]$. Compared with placebo, performance on this factor was significantly improved by Cereboost ${ }^{\mathrm{TM}}$ at $3 \mathrm{~h}(p=0.017)$ with a trend towards improved performance at $6 \mathrm{~h}$ $(p=0.08)$ post treatment (Table 3 and Figure 1).

Inspection of the individual task outcomes contribution to the working memory factor showed that there was a significant Treatment $\times$ Time interaction $[F(2,86)=3.891, p=0.024]$ on the initial ANOVA (Table 2 and Figure 1) for Spatial Working Memory sensitivity index. Compared with placebo, Cereboost $^{\mathrm{TM}}$ was associated with an improvement on this task at $3 \mathrm{~h}(p=0.022)$ with a trend towards a significant improvement at $6 \mathrm{~h}$ post treatment $(p=0.058)$.

There was no significant Treatment or Treatment $\times$ Time interactions for the secondary memory, speed of memory, accuracy of attention or speed of attention factors.

\section{COMPASS individual tasks outcome measures}

Mean pre-dose baseline raw scores and change from baseline scores, for each treatment at each post-time point on the selected tasks of the COMPASS battery are presented in Table 4 . There were no significant main effects of treatment or any Treatment $\times$ Time interactions for any COMPASS measure.

\section{Mood measures}

None of the mood measures were significantly affected by treatment (Table 5 ).

\section{Blood glucose levels}

According to initial ANOVA $[F(1,52)=0.088$, $p=0.768$ ], blood glucose levels were not affected by the treatment and there were no treatment $\mathrm{x}$ time interactions (Table 6).

Table 6. Effects of P. quinquefolius (Cereboost ${ }^{\mathrm{TM}}$ ) on peripheral levels of blood glucose

\begin{tabular}{|c|c|c|c|c|c|}
\hline Measure & (n) & $\begin{array}{l}\text { Pre-dose } \\
\text { baseline } \\
\text { score }\end{array}$ & $1 \mathrm{~h}$ & $3 \mathrm{~h}$ & $6 \mathrm{~h}$ \\
\hline \multicolumn{6}{|l|}{$\begin{array}{l}\text { Blood glucose levels } \\
\text { (mmol/litre) }\end{array}$} \\
\hline Placebo & 52 & $6.56(1.32)$ & $-1.73(1.58)$ & $-0.31(1.67)$ & $-2.04(1.37)$ \\
\hline P. quinquefolius & & $6.54(1.19)$ & $-1.65(1.26)$ & $-0.33(1.28)$ & $-1.87(1.71)$ \\
\hline
\end{tabular}

Mean baseline and change from baseline scores are presented with standard deviations in parentheses. 


\section{Tolerability}

The product was well tolerated. One AE was recorded during the study, and was classified as remotely related to the study product.

\section{DISCUSSION}

This randomised, crossover, placebo-controlled study investigated the acute cognitive, mood and glucoregulatory effects of a single $(200 \mathrm{mg}$ ) dose of P.quinquefolius (Cereboost $^{\mathrm{TM}}$ ) in middle-aged (40-60years) volunteers. Supporting our hypothesis, Cereboost ${ }^{\mathrm{TM}}$ improved working memory performance as indexed by the working memory factor score of the CDR test battery. This effect was time dependent, with significant improvement observed at $3 \mathrm{~h}$ and a trend towards improvement at $6 \mathrm{~h}$ post treatment. Whilst a working memory factor encompassed accuracy indices (SI) of both spatial and numeric working memory, improvement was largely driven contributed by the spatial working memory task (Figure 1). The current study demonstrated that a single dose of $200 \mathrm{mg}$ of Cereboost ${ }^{\mathrm{TM}}$ elicited modulation of cognitive performance of healthy middle-aged adults in the absence of mood effects.

These results partially replicate previously reported effects of Cereboost ${ }^{\mathrm{TM}}$ on cognitive functions in a younger population, with treatment-related improvements mainly observed within the cluster of the tasks targeting working memory (Scholey et al., 2010). The previous randomised, double-blind, placebocontrolled, crossover trial examined the acute mood, neurocognitive and glycaemic effects of three doses $(100,200$ and $400 \mathrm{mg})$ of P.quinquefolius in healthy young adults (mean age $=25.2$ years, \pm 4.97 ). Like here, there was a significant improvement of working memory performance associated with $P$. quinquefolius. The middle $200 \mathrm{mg}$ dose differentially improved speed of numeric working memory. Both the lower $(100 \mathrm{mg})$ and the higher $(400 \mathrm{mg}$ ) doses enhanced alphabetic working memory speed at all time points. In addition, all three doses significantly improved Corsi blocks performance at all testing time points. Counter to predictions, the $200 \mathrm{mg}$ of P.quinquefolius did not improve performance of Corsi blocks and alphabetic working memory in the older middle-aged cohort. However, differences in populations (middle-aged vs. young adults) may be partially accountable for the observed differences. Another possible explanation could be the structure of the testing days. In the current study, participants attended the laboratory for approximately $8.5 \mathrm{~h}$, almost $2 \mathrm{~h}$ longer than the previous study. Extending length of the study may have induced fatigue. The Corsi block tasks came relatively late in a longer testing session than in the previous study in the young cohort. It is possible that participants expended cognitive and/or attentional resources on other tasks leading to a null effect on Corsi blocks.

The relatively high levels of ginsenoside $R b_{1}$ in Cereboost ${ }^{\mathrm{TM}}$ P. quinquefolius may be partially responsible for its effect on cognition. According to Hasegawa (2004) and Tawab et al. (2003) ginsenosides, panaxadiols, and $\mathrm{Rb}_{1}$ in particular, can be quickly absorbed from the gastrointestinal system and remain in the parent form after entering systemic circulation for hours. However, these compounds may also undergo a process of stepwise glycosylation facilitated by intestinal flora reaching systemic circulation as their degradation products (compound-K (M1), $\mathrm{M} 4, \mathrm{Rh}_{1}$ and $\mathrm{F} 1$ ), where they are esterified to fatty acid conjugates remaining active longer than the parent compound. Indeed, it has been demonstrated that intestinal bacterial metabolic activity varies amongst individuals, and activity of orally administered ginsenosides correlates with the ginsenosidehydrolyzing potential of intestinal flora (Lee et al., 2009). As the deglycosylation process is crucial for pharmacological expression of ginsenosides, individual differences in bacterial ginsenoside-hydrolysing potential and gastric efficiency determined by various conditions including diet, health and stress levels may lead to different levels of ginseng efficacy (Hasegawa, 2004). Hence, it is possible that gastrointestinal efficiency of the middle-aged group or this particular group of volunteers differed from that of the participants recruited for the previous study, which determined differences in effects of ginsenosides on cognitive function and mood.

Choice of the single dose $(200 \mathrm{mg})$ for investigating cognitive and mood effects of Cereboost ${ }^{\mathrm{TM}}$ in middleaged cohort might have been another reason for discrepancies between the findings of the two studies. Some of the cognitive measures, such as choice reaction time and alphabetic working memory speed, were improved by the lowest $(100 \mathrm{mg})$ and the highest $(400 \mathrm{mg})$ doses in the younger cohort; therefore, assessment of these doses might have revealed a greater scope of benefits for cognition in middle-aged population. Therefore, implementation of the different dosages of the extract is required to further understanding of its effects in middle-aged adults.

Findings of the current study established that the ingestion of single $200 \mathrm{mg}$ dose of Cereboost ${ }^{\mathrm{TM}}$ does not modulate blood glucose in middle-aged healthy 
volunteers, with this pattern being consistent with outcomes of the preceding study, which investigated glucoregulatory effects of the same extract in a younger (18-40 years) age group (Scholey et al., 2010). This is despite the fact that the cohort was older than in our previous study (Scholey et al., 2010). This suggests that P.quinquefolius may not acutely modulate blood glucose levels despite the fact that the study was strictly controlled for glycaemic parameters. As there were no changes in blood glucose in either study, it seems unlikely that $P$. quinquefolius exerts mediating effects on cognitive function through glucose or insulin-mediated mechanisms.

Vuksan et al. (2000b) previously reported that $P$. quinquefolius root extract attenuated postprandial blood glucose when co-administered with $25 \mathrm{~g}$ of glucose in both healthy and diabetic volunteers; however, hypoglycaemic effects somehow differed between two groups. Prominent hypoglycaemic effects of $P$. quinquefolius were observed in diabetic population regardless of a dose of the herb or a time of its administration in relation to the glucose load. In contrast, a different profile of treatment-related activity emerged in healthy volunteers: it was effective when administered $40 \mathrm{~min}$ prior to a glucose challenge and in fasting conditions only. In relation to the current study, participants were required to fast prior to the sessions in order to control for consumption of standard breakfast in both treatment conditions, they were provided with food; therefore, implementation of fasting regiments may reveal a completely different pattern of glucoregulatory changes in response to $P$. quinquefolius.

Whilst the majority of studies have focused on the acute postprandial effects of P.quinquefolius alone or when administered in conjunction with a glucose load, other studies have attempted to delineate chronic effects of the herb in humans (Vuksan et al., 2001; Vuksan and Sievenpiper, 2005). Administration of $P$. quinquefolius $(1 \mathrm{~g})$ three times daily (40 min before meals) to 24 subjects diagnosed with Type 2 Diabetes mellitus for 8 weeks resulted in significant reduction of fasting blood glucose and drop in levels of $\mathrm{HbA} 1 \mathrm{c}$ accompanied by observable but insignificant increase of insulin levels suggestive of improved pancreatic $\beta$-cell function. Vuksan et al. (2001) offered an explanation that $P$.quinquefolius glucoregulatory efficacy may be related to modulation of the post-absorptive early phase of insulin secretion, the loss of which marks Type 2 Diabetes mellitus. Noteworthy, although $P$. quinquefolius generally tends to lower postprandial blood glucose, its extract with depressed ginsenoside profile was not effective (Sievenpiper et al., 2004), highlighting the significance of ginsenoside expression in P.quinquefolius extracts for glucoregulation. The outcomes of another study into the glucoregulatory effects of different ginseng types by Sievenpiper et al. (2004) indicated that the ratio of panaxadiols/panaxatriols is inversely correlated with glucose-lowering efficacy of ginsengs, which may explain differential potency of various extracts. Investigation of the glucoregulatory effects of Cereboost ${ }^{\mathrm{TM}}$ in populations with glucoregulatory deficiencies and in chronic regiments may help to specify its glucoregulatory properties.

The underlying neurocognitive mechanisms underpinning the effects of P.quinquefolius on working memory in healthy young and middle-aged adults remain to be established. To date, no published studies have investigated the effects of P.quinquefolius on brain bioactivity. However, Kennedy et al. (2003) reported acute effects of standardised extract of $P$. ginseng (G115) on brain electrical activity in 15 healthy young (mean age 26.6) volunteers. This double-blind, placebo-controlled, balanced crossover study implemented a standard auditory oddball paradigm when participants were paying attention to the infrequent target tones presented randomly amongst frequent non-target tones. Administration of P.ginseng $(200 \mathrm{mg})$ initiated significant reduction of P300 latency of the auditory evoked response potential across left temporal and occipital regions without exerting changes in its amplitude. Additionally, P. ginseng modulated cerebral EEG activity registered in 'eyes closed' condition across a number of frequency bands. $P$. ginseng facilitated overall reduction in theta, beta and alpha frequency predominantly observed at the frontal sites. Authors suggested that these effects may be associated with ability of ginsenosides to modulate EEG activity through cholinergic neurotransmitter systems or their ability to increase cerebral blood flow hence intensify delivery of metabolic substrates to cerebral structures. Whilst the study provides evidence that P.ginseng facilitates measurable neurocognitive changes in humans, these results are unlikely to be pertinent to the current investigation of P.quinquefolius, as the profile of ginsenosides in Cereboost ${ }^{\mathrm{TM}}$ differs from that of $P$. ginseng extract G115 with respect to individual ginsenoside content, concentrations and the ratio of panaxadiols and panaxatriols. For example, levels of $\mathrm{Rb} 1, \mathrm{Rd}$ and $\mathrm{Re}$ ginsenosides in Cereboost ${ }^{\mathrm{TM}}$ is comparatively higher than that of G115 therefore pharmacological profiles of the extracts are likely to differ. Differences between the acute behavioural effects of P.quinquefolius and $P$.ginseng are slowly emerging facilitated by the 
availability of the standardised extracts Cereboost ${ }^{\mathrm{TM}}$ and G115 and become evident from the broad evaluation of the studies based on robust double-blind, repeated-measures methodology with the use of the computerised cognitive and mood assessments.

Behavioural effects of P.quinquefolius reported by Scholey et al. (2010) and observed in the current investigation differ from the acute effects of $P$. ginseng (G115) outlined in the behavioural studies of Kennedy et al. (2001b; 2002). Briefly, P. ginseng was also capable of enhancing memory performance at levels that are comparable with pharmaceutical cognitive enhancers (Neale et al., 2013). Improvements were largely associated, however, with episodic or 'secondary' memory with changes occurring up to $6 \mathrm{~h}$ postdose in response to the intake of $400 \mathrm{mg}$ dose (Kennedy et al., 2001b; 2002). In contrast, the current research suggests that cognitive effects of $P$. quinquefolius are differentially associated with working memory processes.

The mechanisms behind facilitation of cognitive performance by Cereboost ${ }^{\mathrm{TM}}$ in both young and middle-aged volunteers are unlikely to be associated with a single physiological process. Central cholinergic systems have been implicated in mediating learning and memory processes (Perry, 1986) and cholinergic effects of isolated ginsenosides have been revealed in animal models. For example, $\mathrm{Rb}_{1}$ increased expression of choline acetyltransferase and nerve growth factor messenger RNA (Salim et al., 1997), stimulated synaptosomal choline uptake and acetylcholine release (Benishin et al., 1991; Benishin, 1992) and modulated long-term potentiation in the rat hippocampal formation (Abe et al., 1994). Results of several animal studies show that $\mathrm{Rb}_{1}$ (Benishin et al., 1991), Rg1 (Yamaguchi et al., 1995) and $\mathrm{Re}$ (Yamaguchi et al., 1996) prevent scopolamineinduced memory deficits, by increasing cholinergic activity. Future studies should elucidate the mechanisms of the observed effects of Cereboost ${ }^{\mathrm{TM}}$ on working memory via, for instance, scopolamine challenge in humans.

Furthermore, we cannot rule out the possibility of ginsenoside effects on other neurotransmitter systems (Kennedy and Scholey, 2003). Because cognitive improvements are most consistently found in working memory measures, one plausible explanation could be ginsenoside-mediated augmentation of the dopaminergic neurotransmitter system (Ellis and Nathan, 2001). On the other hand, considering evidence of the cholinergic contribution to these processes (Rusted and Warburton, 1989), and a relatively high content of ginsenoside $R b_{1}$, possessing potent cholinergic effects
(Benishin et al., 1991) in Cereboost ${ }^{\mathrm{TM}}$ P. quinquefolius, interaction of ginsenosides with cholinergic systems may contribute to the observed cognitive effects.

In addition, capacity of ginsenosides to modulate nitric oxide production has been previously demonstrated (Smith et al., 2014). Considering that nitric oxide enhances endothelial function and modulates cerebral blood flow, efficient delivery of metabolic substrates to the active cortical sites could be one of the potential mechanisms underlying cognitive improvements exerted by ginseng species. Additional measurements of cardiovascular function such as registration of cerebral blood flow of the participants in Cereboost $^{\mathrm{TM}}$ and placebo conditions may provide useful insight into these mechanisms.

In summary, the present results confirm that Cereboost $^{\mathrm{TM}}$ has the ability to positively modulate working memory in middle-aged adults, predominantly $3 \mathrm{~h}$ following administration. These results are broadly consistent with previous work in younger adults (Scholey et al., 2010) where working memory was also improved. The mechanism of action remains unknown, but it is unlikely that regulation of blood glucose by P.quinquefolius is involved. Further research is required in order to evaluate the effects of Cereboost $^{\mathrm{TM}}$ at different dosages in this and other populations.

\section{CONFLICT OF INTEREST}

Scholey has received funds for travel, speaking engagements and consultancy from Naturex. Meyer, Roller and Ibarra are or have been employed by Naturex. Scholey, Wesnes and Pipingas have received research funding, hospitality, speaker's fees and consultancy from the supplement industry.

\section{ACKNOWLEDGEMENTS}

This work was sponsored by a grant from Naturex.

\section{REFERENCES}

Alberti KGMM, Zimmet PE. 1998. Definition, diagnosis and classification of diabetes mellitus and its complications. Part 1: diagnosis and classification of diabetes mellitus. Provisional report of a WHO consultation. Diabetic Med 15: 539-553.

Abe K, Cho SI, Kitagawa I, Nishiyama N, Saito H. 1994. Differential effects of ginsenoside Rb1 and malonylginsenoside Rb1 on long-term potentiation in the dentate gyrus of rats. Brain Res 649: 7-11.

Attele AS, Wu JA, Yuan CS. 1999. Ginseng pharmacology: multiple constituents and multiple actions. Biochem Pharmacol 58: 1685-1693.

Benishin CG. 1992. Actions of ginsenoside Rb1 on choline uptake in central cholinergic nerve endings. Neurochem Int 21: 1-5.

Benishin CG, Lee R, Wang LC, Liu HJ. 1991. Effects of ginsenoside Rb1 on central cholinergic metabolism. Pharmacology 42(4): 223-229. 
Bishop NA, Lu T, Yankner BA. 2010. Neural mechanisms of ageing and cognitive decline. Nature 464: 529-535.

Bond A, Lader M. 1974. The use of analogue scales in rating subjective feelings. Br J Med Psychol 47: 211-218.

Ellis KA, Nathan PG. 2001. The pharmacology of human working memory. Int J Hum Psychopharmacol 4: 299-313.

Folstein M. 2007. Improving dementia assessment by reducing sample heterogeneity. Int Psychogeriatr 19: 383-389.

Folstein MF, Folstein SE, McHugh PR. 1975. Mini-mental state. A practical method for grading the cognitive state of patients for the clinician. $J$ Psychiatr Res 12: 189-198.

Harkey MR, Henderson GL, Gershwin ME, Stern JS, Hackman RM. 2001. Variability in commercial ginseng products: an analysis of 25 preparations. Am J Clin Nutr 73: 1101-1106.

Haskell CF, Kennedy DO, Wesnes KA, Milne AL, Scholey AB. 2007. A double-blind, placebo-controlled, multi-dose evaluation of the acute behavioural effects of guarana in humans. $J$ Psychopharmacol 21: 65-70.

Hasegawa H. 2004. Proof of the mysterious efficacy of ginseng: basic and clinical trials: metabolic activation of ginsenoside: deglycosylation by intestinal bacteria and esterification with fatty acids. J Pharmacol Sci 95: 153-157.

Kennedy D, Scholey A, Drewery L, Marsh R, Moore B, Ashton H. 2003. Electroencephalograph effects of single doses of Ginkgo biloba and Panax ginseng in healthy young volunteers. Pharmacol Biochem Behav 75: 701-709.

Kennedy DO, Jackson PA, Haskell CF, Scholey AB. 2007. Modulation of cognitive performance following single doses of $120 \mathrm{mg}$ Ginkgo biloba extract administered to healthy young volunteers. Hum Psychopharmacol 22: 559-566.

Kennedy DO, Scholey AB. 2003. Ginseng: potential for the enhancement of cognitive performance and mood. Pharmacol Biochem Behav 75: 687-700.

Kennedy DO, Scholey AB, Wesnes KA. 2000. The dose-dependent cognitive effects of acute administration of Ginkgo biloba to healthy young volunteers. Psychopharmacology (Berl) 151: 416-423.

Kennedy DO, Scholey AB, Wesnes KA. 2001a. Differential, dose dependent changes in cognitive performance following acute administration of a Ginkgo biloba/Panax ginseng combination to healthy young volunteers. Nutr Neurosci 4: 399-412.

Kennedy DO, Scholey AB, Wesnes KA. 2001b. Dose dependent changes in cognitive performance and mood following acute administration of Ginseng to healthy young volunteers. Nutr Neurosci 4: 295-310.

Kennedy DO, Scholey AB, Wesnes KA. 2002. Modulation of cognition and mood following administration of single doses of Ginkgo biloba, ginseng, and a ginkgo/ginseng combination to healthy young adults. Physiol Behav 75: 739-751.

Lee J, Lee E, Kim D, Lee J, Yoo J, Koh B. 2009. Studies of absorption, distribution and metabolism of ginseng in humans after oral administration. J Ethnopharmacol 122: 143-148.

Lovibond PF, Lovibond SH. 1995. The structure of negative emotional states: comparison of the Depression Anxiety Stress Scales (DASS) with the Beck Depression and Anxiety Inventories. Behav Res Ther 33: $335-343$.

Macpherson H, Roberstson B, Sünram-Lea S, Stough C, Kennedy D, Scholey A. 2014. Glucose administration and cognitive function: differential effects of age and effort during a dual task paradigm in younger and older adults. Psychopharmacology (Berl). in press. DOI: 10.1007/ s00213-014-3750-8

Matthews DR, Holman RR, Bown E, et al. 1987. Pen-sized digital 30-second blood glucose meter. Lancet 1: 778-779.

Messier C, Tsiakas M, Gagnon M, Desrochers A. 2010. Effect of age and glucoregulation on cognitive performance. J Clin Exp Neuropsychol 32: $809-821$.

Neale C, Camfield D, Reay J, Stough C, Scholey A 2013. Cognitive effects of two nutraceuticals Ginseng and Bacopa benchmarked against modafinil: a review and comparison of effect sizes. Brit $J$ Clin Pharmacol 75: 728-737.

Pengelly A, Snow J, Mills SY, Scholey A, Wesnes K, Butler LR. 2012. Short-term study on the effects of rosemary on cognitive function in an elderly population. J Med Food 15: 10-17.
Perry EK. 1986. The cholinergic hypothesis--ten years on. Br Med Bull 42: 63-69.

Price CP, Koller PU. 1988. A multicentre study of the new Reflotron system for the measurement of urea, glucose, triacylglycerols, cholesterol, gamma-glutamyltransferase and haemoglobin. J Clin Chem Clin Biochem 26: 233-250.

Qi LW, Wang CZ, Yuan CS. 2011. Ginsenosides from American ginseng: chemical and pharmacological diversity. Phytochemistry 72: 689-699.

Reay JL, Kennedy DO, Scholey AB. 2005. Single doses of Panax ginseng (G115) reduce blood glucose levels and improve cognitive performance during sustained mental activity. $J$ Psychopharmacol 19: 357-365.

Reay JL, Kennedy DO, Scholey AB. 2006. Effects of Panax ginseng, consumed with and without glucose, on blood glucose levels and cognitive performance during sustained 'mentally demanding' tasks. $J$ Psychopharmacol 20: 771-781.

Rusted JM, Warburton, DM. 1989. Cognitive models and cholinergic drugs. Neuropsychobiology 21: 31-36.

Salim KN, McEwen BS, Chao HM. 1997. Ginsenoside Rb1 regulates ChAT, NGF and trkA mRNA expression in the rat brain. Mol Brain Res 47(1-2): 177-182.

Scholey AB, Kennedy DO. 2002. Acute, dose-dependent cognitive effects of Ginkgo biloba, Panax ginseng and their combination in healthy young volunteers: differential interactions with cognitive demand. Hum Psychopharmacol 17(1): 35-44.

Scholey AB, Kennedy DO. 2004. Cognitive and physiological effects of an 'energy drink': an evaluation of the whole drink and of glucose, caffeine and herbal flavouring fractions. Psychopharmacology (Berl) 176: $320-330$.

Scholey AB, Kennedy DO, Wesnes K. 2005. The psychopharmacology of herbal extracts: issues and challenges. Psychopharmacology (Berl) 198: 705-707.

Scholey AB, Tildesley NT, Ballard CG, et al. 2008. An extract of Salvia (sage) with anticholinesterase properties improves memory and attention in healthy older volunteers. Psychopharmacology (Berl) 198(1): 127-139.

Scholey A, Ossoukhova A, Owen L, et al. 2010. Effects of American ginseng (Panax quinquefolius) on neurocognitive function: an acute, randomised, double-blind, placebo-controlled, crossover study. Psychopharmacology (Berl) 212: 345-356.

Sievenpiper JL, Arnason JT, Vidgen E, Leiter LA, Vuksan V. 2004. A Systematic Quantitative Analysis of the Literature of the High Variability in Ginseng (Panax spp.) Should ginseng be trusted in diabetes? Diabetes Care 27: 839-840.

Smith I, Williamson EM, Putnam S, Farrimond J, Whalley BJ. 2014. Effects and mechanisms of ginseng and ginsenosides on cognition. Nutr Rev 72: 319-333.

Stough C, Clarke J, Lloyd J, Nathan PJ. 2001. Neuropsychological changes after 30-day Ginkgo biloba administration in healthy participants. Int $J$ Neuropsychopharmacol 4: 131-134.

Sunram-Lea S, Birchall R, Wesnes K, Petrini O. 2005. The effect of acute administration of $400 \mathrm{mg}$ of Panax ginseng on cognitive performance and mood in healthy young volunteers. Curr Top Neutraceutical Res 3: 65-74.

Tawab M, Bahr U, Karas M, Wurglics M, Schubert-Zsilavecz M. 2003. Degradation of ginsenosides in humans after oral administration. Drug Metab Disposit 31: 1065-1071.

Vuksan V, Stavro MP, Sievenpiper JL, et al. 2000a. Similar postprandial glycemic reductions with escalation of dose and administration time of American ginseng in type 2 diabetes. Diabetes Care 23: 1221-1226.

Vuksan V, Stavro MP, Sievenpiper JL, et al. 2000b. American ginseng improves glycemia in individuals with normal glucose tolerance: effect of dose and time escalation. $J$ Am Coll Nutr 19: 738-744.

Vuksan V, Sievenpiper JL, Koo VYY, et al. 2000c. American Ginseng (Panax quinquefolius L) Reduces Postprandial Glycemia in Nondiabetic Subjects and Subjects With Type 2 Diabetes Mellitus. Arch Intern Med 160: 1009-1013.

Vuksan V, Sievenpiper JL, Wong J, Xu Z, Beljan-Zdravkovic U, Arnason JT, Francis T. 2001. American ginseng (Panax quinquefolius L.) attenuates postprandial glycemia in a time-dependent but not dose-dependent manner in healthy individuals. Am J Clin Nutr 73: 753-758. 
Vuksan V, Sievenpiper JL. 2005. Herbal remedies in the management of diabetes: lessons learned from the study of ginseng. Nutr Metab Cardiovascular Dis 15: 149-160.

Wesnes KA, Ward T, McGinty A, Petrini O. 2000. The memory enhancing effects of a Ginkgo biloba/Panax ginseng combination in healthy middle-aged volunteers. Psychopharmacology (Berl) 152: 353-361.
Yamaguchi Y, Haruta K, Kobayashi H. 1995. Effects of ginsenosides on impaired performance induced in the rat by scopolamine in a radialarm maze. Psychoneuroendocrinol 20: 645-653.

Yamaguchi Y, Higashi M, Kobayashi H. 1996. Effects of ginsenosides on impaired performance caused by scopolamine in rats. Eur J Pharmacol 312: $149-151$. 